\title{
Dynamics of a Bose-Einstein condensate at finite temperature in an atomoptical coherence filter
}

\author{
F. Ferlaino, P. Maddalonit S. Burger, F. S. Cataliotti, C. Fort, M. Modugno, and M. Inguscio \\ INFM-European Laboratory for Nonlinear Spectroscopy (LENS) and Dipartimento di Fisica, Università di Firenze, \\ L.go E. Fermi 2, I-50125 Firenze, Italy
}

(November 7, 2018)

The macroscopic coherent tunneling through the barriers of a periodic potential is used as an atomoptical filter to separate the condensate and the thermal components of a ${ }^{87} \mathrm{Rb}$ mixed cloud. We condense in the combined potential of a laser standing-wave superimposed on the axis of a cigar-shape magnetic trap and induce condensate dipole oscillation in the presence of a static thermal component. The oscillation is damped due to interaction with the thermal fraction and we investigate the role played by the periodic potential in the damping process.

PACS numbers: 03.75.Fi, 32.80.Pj, 03.75.-b

The combination of Bose-Einstein condensates (BECs) with periodic optical potentials provides a versatile tool for getting a deeper understanding of macroscopic quantum phenomena, directly addressing the issue of phase coherence between degenerate coupled Bose gases [1 [4] recently culminating in the observation of the Mott insulator phase transition [5]. Properties of condensates confined in an optical lattice have opened new perspectives in the investigation of different dynamical phenomena such as superfluid flow [6], atomic Bloch oscillations [7], the Josephson effect [8] and have stimulated speculations on a wide range of phenomena including solitons [9], dynamical instability [10] and transport behavior [11].

The dynamics of BECs at finite temperatures, is a challenging question even in the pure harmonic case. Many efforts have been devoted to the extension of theoretical models at nonzero temperature and to the study of the low-energy collective excitations in presence of a significant thermal component [12 177.

From the experimental point of view the effects of the interactions between the condensate and the thermal fraction on the modes of excitation have been investigated only in harmonic potentials. Temperature dependence of damping and frequency shifts in quadrupole and scissors modes have been investigated in a TOP trap [18, 19 while also a damped out-of-phase dipolar oscillation of the thermal cloud and the condensate has been observed in a cloverleaf trap [20].

In this Letter we address the problem of the dynamics of a condensate in a periodic potential at finite temperature. This is done by using an optical lattice as an atomoptical filter to induce, through a selective manipulation, a relative motion between the condensate and thermal components of a magnetically trapped mixed cloud of rubidium atoms. In the presence of the periodic potential these two components respond differently to a sudden displacement of the magnetic trap. Atoms with sufficiently long coherence lengths are allowed to tunnel through the potential barriers of a one dimensional optical lattice while atoms with a higher kinetic energy remain confined in the potential wells. While the condensate coherently flows through the optical barriers, thus performing a dipole oscillation, the thermal component is blocked in the presence of the periodic potential and provides a damping mechanism. In particular, we observe a sharp change of the damping rate in the BEC dipole oscillations by increasing the optical potential depth.

Our procedure for creating Bose condensates in an optical lattice, already described in details elsewhere [6], can be summarized as follows. Starting with a sample of ultracold atoms of ${ }^{87} \mathrm{Rb}$ confined in a Ioffe-type magnetic trap in the $\left(F=1, m_{F}=-1\right)$ state, we perform rfevaporative cooling until we reach a temperature slightly above the condensation threshold. Next, we superimpose a one-dimensional optical lattice to the longitudinal $x$-axis of the magnetic trap by means of a far detuned, retroreflected laser beam. We then continue the evaporation process through the phase-transition temperature [21]. In this way, the atomic cloud is prepared in an equilibrium state of the combined magnetic and optical dipole potentials. In this experiment we typically stop the evaporation when the fraction of condensed atoms is $\simeq 20 \%$ corresponding to a temperature $T \simeq 120 \mathrm{nK}$. In the atomic cloud region the resulting potential has the form

$$
\begin{aligned}
V & =V_{\text {mag }}+V_{\text {opt }} \\
& =\frac{1}{2} m\left[\omega_{x}^{2} x^{2}+\omega_{\perp}^{2}\left(x^{2}+y^{2}\right)\right]+s E_{R} \cos ^{2}\left(\frac{2 \pi x}{\lambda}\right)
\end{aligned}
$$

with $m$ the atomic mass, $\omega_{x}=2 \pi \times 9 \mathrm{~Hz}$ and $\omega_{\perp}=$ $2 \pi \times 92 \mathrm{~Hz}$ the axial and radial frequency of the magnetic potential respectively. In (1) the optical potential depth is expressed, via the dimensionless factor $s$, in units of the recoil energy of an atom absorbing one laser photon, $E_{R}=h^{2} / 2 m \lambda^{2}$, where $\lambda$ is the wavelength of the laser creating the standing wave.

By varying the intensity of the laser beam, (detuned typically $\Delta \lambda \simeq 3 \mathrm{~nm}$ to the blue of the $D_{1}$ line at $\lambda \simeq 795 \mathrm{~nm})$, we change the value of $s$ up to 2.5 . The optical potential is then calibrated by measuring the 
Rabi frequency of the Bragg transition between the momentum states $-h / \lambda$ and $+h / \lambda$ induced by the standing wave 22. Due to the large detuning of the optical lattice, the maximum spontaneous scattering rate is $\Gamma_{s p}=0.25 \mathrm{~Hz}$ and spontaneous scattering can be neglected on the timescale of our experiment.

In order to induce the relative center-of-mass motion of the condensate and the thermal fractions in the combined trap, we first displace instantaneously $\left(t_{d i s} \ll 2 \pi / \omega_{x}\right)$ the magnetic trapping potential in the $x$-direction by a distance $\Delta x=18 \mu \mathrm{m}$ sufficiently small to remain in the superfluid regime [6]. After the displacement, the atomic cloud finds itself out of equilibrium, nevertheless due to the presence of the optical lattice, only the condensate is forced into motion by the magnetic potential gradient. Indeed, when the potential energy given by the displacement is smaller than the optical potential depth, as in our experiment, a motion along the $x$-direction can be established only by means of tunneling through the barriers of the standing wave. We have previously shown [8] that a pure condensate and a thermal cloud above $\mathrm{T}_{c}$ behave in a dramatically different way. The coherence properties of the condensate imply a macroscopic tunneling and a collective dipole motion can occur. On the contrary, the incoherent nature of the thermal cloud drastically reduces the collective tunneling probability, thus preventing the establishing of a collective oscillation. In the present work we produce mixed clouds and the optical lattice acts as a filter to separate the coherent and incoherent components. After the displacement, we wait half a period of the BEC oscillation in the harmonic trap $\left(\pi / \omega_{x}\right)$ then we move back the magnetic potential by the distance $-\Delta x$. At the end of this procedure, the thermal cloud finds itself at rest in the center of the trap, while the condensate oscillates inside the thermal cloud with an initial amplitude $2 \Delta x$. After a variable evolution time, the trapping potential is switched off and the cloud is imaged subsequently to an additional ballistic expansion of $28 \mathrm{~ms}$. From the observed column density we deduce the condensate fraction, the temperature and the centerof-mass position of both the condensate and the thermal components in the trap. In Fig. 1 we report three absorption images corresponding to successive times of the evolution in the combined trap.

In order to show the effects of the optical lattice on the dynamics of a mixed cloud, we study the center-of-mass motion of the condensate and the thermal components in two different configurations. In one (harmonic case) we switch off the periodic potential after the preparatory procedure described above while in the other (combined case) we leave the lattice on also during the subsequent evolution.

Harmonic case - In absence of the lattice both the condensate and the thermal cloud are free to oscillate. Starting from an off-equilibrium configuration in which the thermal cloud is at the center of the trap, the condensate mean-field pushes the thermal fraction out of the potential minimum. During the time evolution the os- cillation amplitude of the thermal cloud rises until an equilibrium is reached (Fig. 2a). Notice that, in a pure harmonic trap, when no relative motion is induced, the BEC dipolar mode can not be affected by the presence of the thermal component 23]. We expect that the kinetic energy lost by the condensate is essentially converted into kinetic energy of the thermal cloud. From the fit of the relative center-of-mass position (Fig. 2b) with a damped oscillation, we obtain a frequency of $8.42 \pm 0.04 \mathrm{~Hz}$ which is smaller of roughly $5 \%$ from the measured trapping frequency. This is a consequence of the interaction between the oscillating condensate and the initially stationary thermal cloud and is similar to the observation reported in [20 for an oscillating thermal cloud in presence of an initially stationary condensate.

Combined case - In presence of the optical lattice the thermal cloud stays locked at the center of the trap due to its incoherent nature, while the condensate oscillates as shown in Fig. 3. Again the coupling between the condensate ad the thermal cloud causes damping in the centerof-mass oscillations of the condensate. Here, the kinetic energy lost by the BEC during the evolution cannot be converted into the kinetic energy of the thermal cloud which is kept fixed by the periodic potential and must be therefore partly converted into internal energy of the cloud, partly absorbed by the optical lattice. Note that in this case the pure condensate would perform undamped oscillations [6].

We investigate the damping rate of the condensate center-of-mass motion as a function of the optical potential depth $s E_{R}$ for a fixed trap displacement. The experiment is performed in the collisionless regime where collisions between thermal atoms are negligible. In this regime Landau damping, present when a thermal bath of elementary excitations absorbs quanta of the condensate collective excitations, represents an important mechanism to explain the dynamical behaviour of trapped Bose gases [24].

For temperatures larger than the chemical potential, the Landau damping rate of low-energy excitations in a harmonically trapped Bose-condensed gas can be estimated using the form 23.

$$
\Gamma_{L}=\frac{3 \pi}{8} \frac{k_{B} T a \omega}{\hbar c}
$$

where $c=\hbar \sqrt{4 \pi a n_{0}} / m$ is the sound velocity in the center of the trap, $n_{0}$ being the central density of the condensate, $a$ the $s$-wave scattering length and $\omega$ the frequency of the collective excitation. In our case, for small values of $s$, we can estimate $\Gamma_{L}$ calculating the central density of the condensate in presence of the periodic potential using a variational approach with a gaussian ansatz for the condensate wave-function. For $s=0.7$ and assuming a fixed temperature $T=120 \mathrm{nK}$, we have $\Gamma_{L}=1.6 \mathrm{~Hz}$ in fair agreement with the experimental results.

In Fig. 4 we show the damping rate measured as a function of the optical potential depth. Close to $s=1$ we 
observe a sudden increase of the damping rate. This occurs in coincidence with the formation of the first bound state of the condensate in the lattice [25]. Actually the occurrence of a band structure strongly modifies the energy spectrum. In particular the Landau damping, being a resonant energy transfer, strongly depends on the form of energy spectrum [15] and an adequate treatment would be needed. In addition a theoretical analysis of finite temperature damping of excitations in presence of a periodic potential should also include other damping mechanisms such as inter-component damping recently suggested in [14]. However, at present, a comprehensive theoretical analysis which accounts for all the effects introduced by the presence of the lattice is lacking.

In conclusion, we have demonstrated the operation of an atomoptical filter capable of separating the condensate and the thermal fraction in a mixed atomic cloud across $T_{c}$. This filtering technique could, e. g., be applied to spatially separate the ground state of a finite temperature BEC fully from the thermal component. In this work, we are able to control the relative motion between the center-of-masses of the condensate and of the thermal fraction in a mixed cloud, this enable us to investigate quantitative effects both in the amplitude and in the frequency of the dipole oscillations. Damping is caused by interactions between condensate and the thermal fractions. Novel features are expected in presence of the periodic lattice potential which modifies the energy spectrum of the system. Indeed by changing the optical lattice parameters a sharp rise in the damping rate has been observed when a bound state is likely to be formed.

We are indebted to Francesco Minardi for many useful discussions and for his help in the initial stage of the experiment. We acknowledge Gabriele Ferrari, Sandro Stringari, Stefano Giorgini and Lev Pitaevskii for fruitful discussions, and Maurizio Artoni for careful reading of the manuscript.

This work has been supported by the EU under Contracts No. HPRI-CT 1999-00111 and No. HPRN-CT2000-00125, by the MURST through the PRIN 1999 and 2001 Initiatives and by the INFM Progetto di Ricerca Avanzata "Photon Matter".

* Also: Dipartimento di Fisica, Università di Padova, via F. Marzolo 8, I-35131 Padova, Italy.

[1] B. P. Anderson and M. A. Kasevich, Science 281, 1686 (1998).

[2] C. Orzel, A. K. Tuchman, M. L. Fenselau, M. Yasuda, and M. A. Kasevich, Science 291, 2386 (2001).

[3] M. Greiner, I. Bloch, O. Mandel, T. W. Hänsch, and T. Esslinger, Phys. Rev. Lett. 87, 160405 (2001).

[4] P. Pedri et al., Phys. Rev. Lett. 87, 220401 (2001).

[5] M. Greiner, O. Mandel, T. Esslinger, T. W. Hänsch and
I. Bloch, Nature 415, 39 (2002)

[6] S. Burger et al., Phys. Rev. Lett. 86, 4447 (2001).

[7] O. Morsch, J. H. Müller, M. Cristiani, D. Ciampini, and E. Arimondo, Phys. Rev. Lett. 87, 140402 (2001).

[8] F. S. Cataliotti et al., Science 293, 843 (2001).

[9] O. Zobay, S. Pötting, P. Meystre, and E. M. Wright, Phys. Rev. A , 59, 643 (1999); A. Trombettoni, and A. Smerzi, Phys. Rev. Lett. 86, 2353 (2001); J. C Bronski, L. D. Carr, B. Deconinck, and J. N. Kutz, Phys. Rev. Lett. 86, 1402 (2001); ; I. Carusotto, D. Embriaco, and G. C. La Rocca, cond-mat/0111308.

[10] F. K. Abdullaev, B. B. Baizakov, S. A. Darmanyan, V. V. Konotop, M. Salerno, Phys. Rev. A, 64043606 (2001); B. Wu, and Q. Niu, Phys. Rev. A Rapid Comm. 64, 061603 (2001).

[11] D. I. Choi, and Q. Niu, Phys. Rev. Lett. 82, 2022 (1999).

[12] D. A. W. Hutchinson, E. Zaremba, and A. Griffin, Phys. Rev. Lett. 78, 1842 (1997).

[13] S. Giorgini, Phys. Rev. A 57, 2949 (1998).

[14] J. E. Williams, and A. Griffin, Phys. Rev. A 63, 23612 (2001); J. E. Williams, and A. Griffin, Phys. Rev. A 64, 013606 (2001).

[15] P. O. Fedichev, G. V. Shlyapnikov, and J. T. M. Walraven, Phys. Rev. Lett. 80, 2269 (1998); W. V. Liu, and W. C. Schieve, e-print cond-mat/9702122.

[16] E. Zaremba, A. Griffin, and T. Nikuni, Phys. Rev. A 57, 4695 (1998).

[17] B. Jackson, and C. S. Adams, Phys. Rev. A 63, 053606 (2001).

[18] D. S. Jin, M. R. Matthews, J. R. Ensher, C. E. Wieman, and E. A. Cornell, Phys. Rev. Lett. 78, 764 (1997).

[19] O. Maragò, G. Hechenblaikner, E. Hodby, and C. Foot, Phys. Rev. Lett. 86, 3938 (2001).

[20] D. M. Stamper-Kurn, H. J. Miesner, S. Inouye, M. R. Andrews, and W. Ketterle, Phys. Rev. Lett. 81, 500 (1998).

[21] The presence of the optical lattice lowers the critical temperature of the system as described in S. Burger, F. S. Cataliotti, C. Fort, P. Maddaloni, F. Minardi, and M. Inguscio, to appear on Europhys. Lett. e-print condmat/0108037.

[22] E. Peik, M. BenDahan, I. Bouchoule, Y. Castin, and C. Salomon, Phys. Rev. A 55, 2989 (1997).

[23] F. Dalfovo, S. Giorgini, S. Stringari e L. P. Pitaevskii, Rev. Mod. Phys. 71,463 (1999).

[24] W. V. Liu, Phys. Rev. Lett. 79, 4056 (1997).

[25] K. Berg-Sørensen, and K. Mølmer, Phys. Rev. A 58, 1480 (1998).

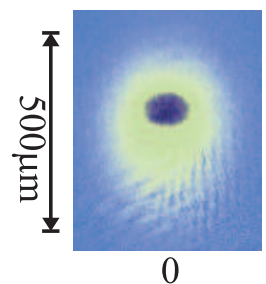

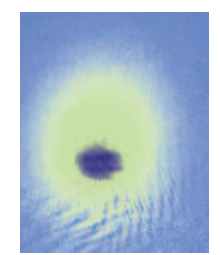

$40 \mathrm{~ms}$

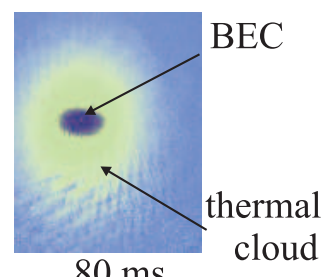

$80 \mathrm{~ms}$
FIG. 1. Absorption images after different evolution times in the combined trap showing the dipole oscillations of the condensate inside the thermal cloud. 

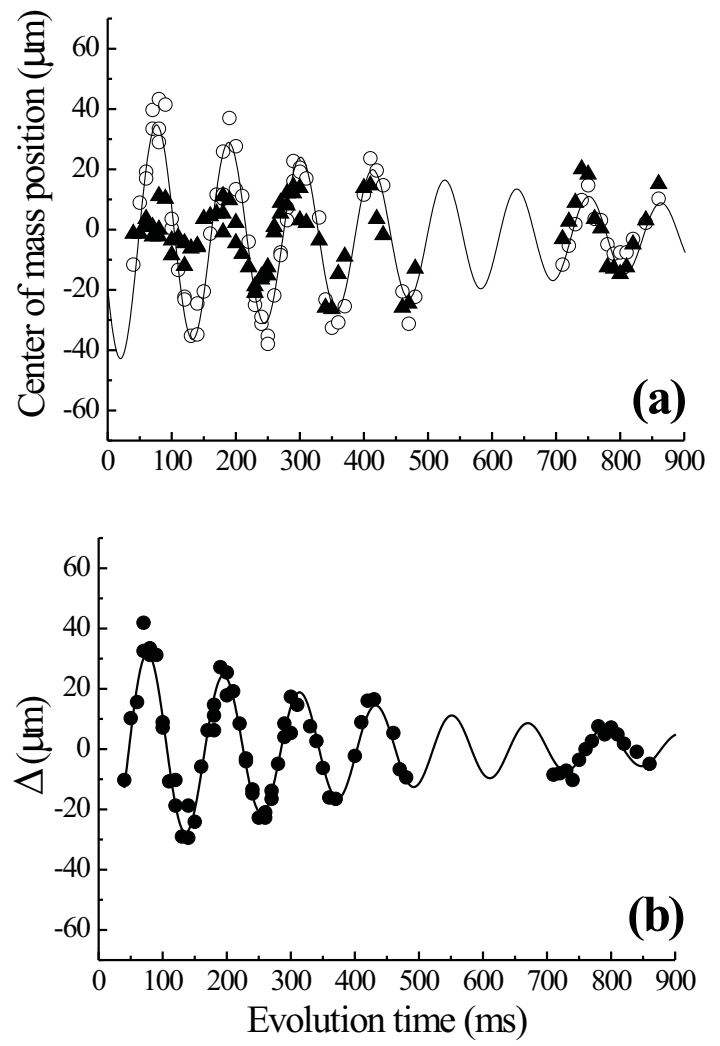

FIG. 2. a) Center of mass position of the condensate (open circles) and the thermal cloud (filled triangles) after the expansion as a function of the time spent in trap when the optical potential is switched off after the initial displacement (the line is a guide to the eye). b) Difference $\Delta$ between the two components center-of-mass position plotted together with a fit to the data (continuous line).

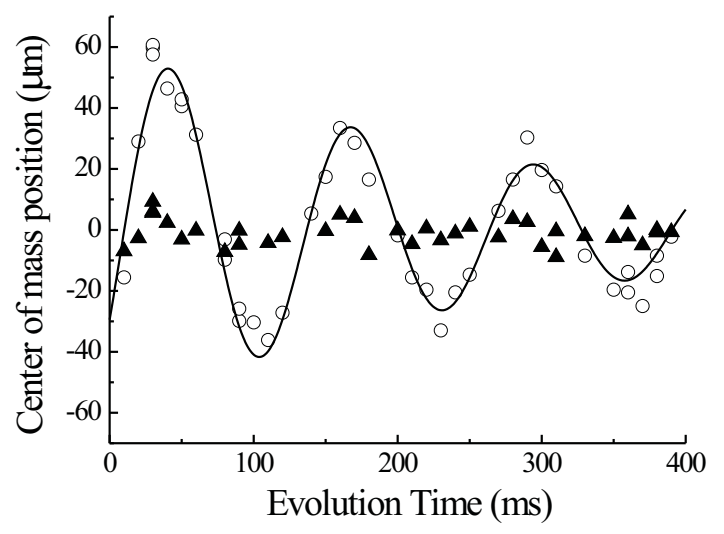

FIG. 3. Center-of-mass position of the condensate (open circles) and the thermal cloud (filled triangles) after the expansion as a function of the time spent in the combined trap $(s=1.8)$ together with a fit to the condensate center-off-mass position (continuous line).

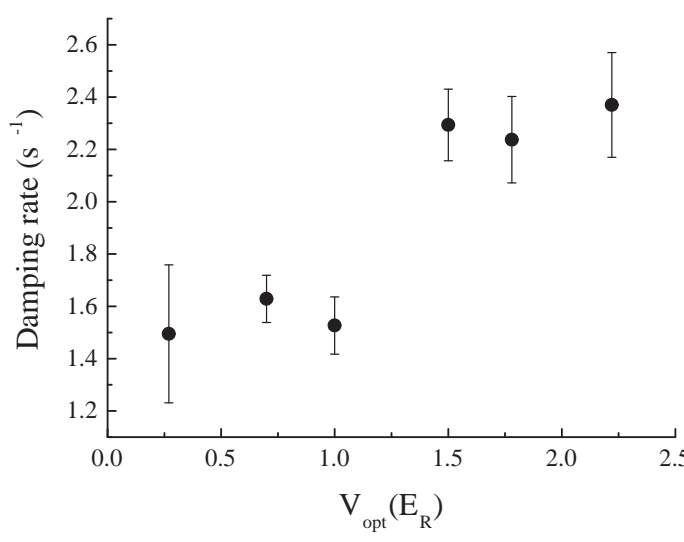

FIG. 4. Damping rate $\Gamma$ as a function of the optical potential depth in units of the recoil energy. The occurrence of a band structure $(s>1)$ is reflected in a jump of the damping rate. 